\title{
Seis Cultivares Potenciales de Papa con Resistencia al Tizón Tardío (Phytophthora infestans) en Bolivia
}

\author{
Enrique Carrasco ${ }^{1}$, Nelson Estrada ${ }^{2}$, Julio Gabriel ${ }^{1}$, Gonzalo Alfaro, ${ }^{3}$ \\ Yvan Larondelle ${ }^{4}$, William García ${ }^{1,}$ Orlando Quiroga ${ }^{1}$
}

\section{Resumen}

El tizón tardío de la papa (Phytophthora infestans) es un problema serio en casi todas las zonas paperas de Bolivia. Esta enfermedad es causante de severas pérdidas en el cultivo de papa, llegando hasta un $100 \%$ si no se aplica ninguna medida de control. Por tanto para un control efectivo del tizón tardío de la papa se requieren numerosas aplicaciones de fungicidas, llegando en algunos casos a más de 10 durante el ciclo de cultivo. Ante este problema el Programa de Investigación de la Papa (PROINPA), ha venido seleccionando durante cinco años clones con resistencia a esta enfermedad provenientes del Centro Internacional de la Papa (CIP) y del Instituto Colombiano Agropecuario (ICA). Por evaluaciones anuales de una población inicial aproximada de 300 clones, se seleccionaron clones por su resistencia al tizón, altos rendimientos y buenos atributos agronómicos. Para evaluar el daño por tizón tardío se usó la escala internacional propuesta por el CIP ( $1=0 \%$ daño a $9=97-100 \%$ daño), que sirvió para calcular el AUDPC (Área Bajo la Curva de Progreso de Phytophthora infestans), que conjuntamente al rendimiento y la calidad culinaria determinaron la selección de seis clones para su entrega como cultivares potenciales, con la activa participación de agricultores y técnicos de las zonas donde fueron conducidos los ensayos. Además se determinaron contenido de nutrientes y de glicoalcaloides de los seis cultivares potenciales, cultivados en dos regiones similares. Se analizó el nivel de glicoalcaloides de la variedad Robusta bajo dos condiciones de cultivo contrastadas

$1 \quad$ M.Sc., Ing. Agr., Tesista. PROINPA (convenio IBTA-CIP-COSUDE). Casilla 4285, Cochabamba, Bolivia.

2 Ph.D. Universidad Nacional de Colombia, Bogotá, Colombia.

3 Ph.D. PAPN - FCyT - Universidad Mayor de San Simón, Cochabamba, Bolivia.

4 Ph.D. BNUT - AGRO- Universidad Católica de Lovaina, Lovaina, Bélgica 
Palabras claves adicionales: clones, escala de evaluación, AUDPC, valor nutricional, glicoalcaloides:

\section{Six Potential Potato Cultivars with Late Blight (Phytophthora infestans) Resistance In Bolivia}

\section{Summary}

Potato late blight (Phytophthora infestans) has become a serious problem all over the potato growing regions of Bolivia. This disease causes great losses in production, affecting up to $100 \%$ of the crop if no control measure is applied. For an effective control of late blight several fungicide applications are needed and in some cases more than ten are required during the entire cultivation cycle.

During the past five years the National Potato Research Program (PROINPA) has been selecting clones with resistance to this disease. These clones were supplied by the International Potato Center (CIP) and the Colombian Agricultural Institute (ICA). From the original population of approximately 300 , clones have been annually evaluated and selected for their late blight resistance, high yield and good agronomic characters. As a result, the six best clones were selected for their performance in relation to the above-mentional factors during five years of evaluation.

In order to measure the damage caused by late blight, the international evaluation scale provided by CIP ( $1=0 \%$ damage to $9=$ 97-100\% damage) was used. Thus, it served to estimate the AUDPC (Area Under Disease Progress Curve), which together with yield and culinary quality determined the selection of six potential varieties with the active participation of farmers and scientists in the areas where testing was performed. Moreover, the nutrient content and the glycoalkaloid level of the clones were determined. The glykoalkaloid level in the variety Robusta, cultivated in two contrasting conditions, was also evaluated.

Additional index words: clones, evaluation scale, AUDPC, glycoalkaloids, nutritive value 
El cultivo de la papa en Bolivia es de suma importancia por constituir un alimento básico en el área rural y urbana. Se cultivan aproximadamente 150,000 ha de papa en el país y en esta actividad están involucradas alrededor de 200,000 familias de agricultores.

Los rendimientos son drásticamente afectados por diversos factores tanto bióticos como abióticos. Dentro de los factores bióticos está el tizón tardío de la papa (Phytophthora infestans (Mont.) De Bary), el cual se presenta en casi todas las zonas paperas de Bolivia comprendidas entre los 2,000 y 3,300 msnm, causando severas pérdidas de hasta un $100 \%$ si no se toma ninguna medida de control y hasta un $60 \%$ con control y en epifitas fuertes. Por tanto para su control se requieren numerosas aplicaciones de fungicidas, llegando en algunos casos a más de 10 durante el ciclo de cultivo. Estas medidas, además de elevar notablemente los costos de producción, dañan el medio ambiente y la salud de los agricultores.

A nivel mundial, se ha venido trabajando durante muchos años en la obtención de cultivares resistentes, aunque en la mayoría de los casos esta resistencia fue vencida al cabo de dos o tres años. En la actualidad existen algunos cultivares que mantienen su resistencia "parcial" por varios años, tal es el caso de los cultivares Monserrate (Colombia), Santa Catalina (Ecuador), Perricholi/Runa Toralapa (Perú/Bolivia), Ackersegen (Alemania), Record, Pimpernel y Libertas (Holanda) y Atzimba (México) (4).

El Programa de Investigación de la Papa, PROINPA, (convenio IBTA-CIPCOSUDE), frente a este grave problema, ha venido generando $y$ seleccionando clones con resistencia al tizón durante los cinco últimos años, con la finalidad de cumplir los siguientes objetivos:

- Obtener cultivares resistentes al tizón de la papa con rendimientos superiores a los cultivares en actual uso y con buenas características agronómicas, culinarias y nutricionales.

- Reducción del número de aplicaciones de fungicidas, favoreciendo de esta manera al medio ambiente, la salud del agricultor y disminuyendo los costos de producción.

- Obtención de cultivares que formen parte de un adecuado "Manejo Integrado" para el control del tizón. 


\section{Materiales y Métodos}

\section{Material experimental}

El material con el que se inició la selección fueron 313 clones, los cuales provenían del Centro Internacional de la Papa (CIP) y del Instituto Colombiano Agropecuario (ICA), incluyendo como testigos cultivares locales como Waych'a (S. tuberosum ssp. andigena) catalogado como tolerante, introducidos como Alpha (S. tuberosum ssp. tuberosum) que se usó como testigo susceptible, además de cultivares comerciales con resistencia al tizón tardío, como es el caso de Rosita (híbrido obtenido en México).

\section{Epocas y localidades}

Los ensayos fueron establecidos en la época de cultivo denominada "siembra grande" que comprende el periodo lluvioso del año (octubremarzo), durante cinco campañas agrícolas (1990-1991 hasta 19941995).

Las localidades se encuentran dentro del Departamento de Cochabamba y en cada campaña se implantaron los ensayos sólo en dos localidades, es decir la campaña 1990/91 y 1991/92 en dos lugares dentro Chullchunqani, 1992/93 Chullchunqani y Candelaria y 1993/94 1994/95 Chullchunqani y Morochata (Tabla 1).

Tabla 1. Localidades donde se evaluaron clones por su resistencia al tizón durante cinco campañas agrícolas.

\begin{tabular}{|lcccc|}
\hline \multicolumn{1}{|c}{ Localidad } & Provincia & $\begin{array}{c}\text { Altitud } \\
\text { (msnm) }\end{array}$ & $\begin{array}{c}\text { Precipitación } \\
\text { (mm)* }\end{array}$ & $\begin{array}{c}\text { Campañas } \\
\text { evaluadas }\end{array}$ \\
\hline Chullchunqani & Carrasco & 3200 & 550 & $1990 / 91-1994 / 95$ \\
Candelaria & Chapare & 2800 & 950 & $1992 / 1993$ \\
Morochata & Ayopaya & 3300 & 900 & $1993 / 94-1994 / 95$ \\
\hline
\end{tabular}

* Precipitación promedio durante el ciclo de cultivo.

\section{Evaluación del material experimental por daño de tizón tardío}

Una vez establecidos los ensayos y luego de haber emergido aproximadamente el $90 \%$ de las plantas, se hizo una aplicación de Dithane M-45 (Mancozeb), en una dosis de $30 \mathrm{~g} / \mathrm{l}$, con el fin de uniformizar los tratamientos antes del inicio del ataque de tizón tardío. 
La evaluación del material genético por daño de tizón se realizó en todos los casos cada siete días, una vez iniciada la infección y durante siete semanas. La evaluación del grado de daño por tizón se hizo utilizando la escala internacional propuesta por el CIP: $1=0 \%$ de daño a $9=97-100 \%$ de daño (3).

A partir de la campaña 1992/93 los clones pasaron a la fase de promisorios, a partir de la cual se procedió a la evaluación con la participación de agricultores y técnicos de otras instituciones que trabajan en la zona; las evaluaciones se realizaron tanto en follaje (grado de daño), como a la cosecha (rendimiento y caracteres agronómicos).

Durante el ciclo de cultivo de la última campaña (94-95) se realizó la caracterización morfológica a nivel de follaje en las dos localidades donde se condujeron los ensayos (Morochata y Chullchunqani).

\section{Evaluación a la cosecha y poscosecha}

A la cosecha que se realiza normalmente en el mes de abril, se evaluaron rendimientos y se procedió a la caracterización morfológica de los tubérculos, además se midió el grado de daño por tizón en tubérculo. Clones con bajo rendimiento, formas indeseables y con daño de tizón fueron descartados.

En la campaña 1994/95, en las pruebas de degustación participaron grupos de 7 a 18 agricultores de cuatro comunidades de la zona de Morochata y de dos comunidades de la zona de Chullchunqani.

Los clones fueron degustados en forma de papa cocida con cáscara, usando como testigo al cultivar Waych'a, catalogado como de alta calidad culinaria.

Para desarrollar este trabajo se consideró una matriz de preferencia (4), en la que los agricultores luego de probar cada trozo de papa (una dividida en cuatro partes), daban la siguiente calificación: 3=bueno, 2=regular y 1=malo, colocando al pie de cada clon degustado granos de maíz, piedrecitas 0 pequeños terrones.

Por otra parte, muestras de los tubérculos de la campaña 94-95 fueron recolectados en las localidades de Morochata y Chullchunqani y luego fueron analizados en laboratorio y por duplicado, tanto enteros como en sólo la parte comestible, es decir estas últimas luego de ser peladas. En cada caso, muestras de 5 tubérculos de 4.5 y $6 \mathrm{~cm}$ de diámetro fueron enviados a los laboratorios de la Universidad Católica de Lovaina (Bélgica). 
También en cada caso, dos de estos tubérculos fueron pelados y liofilizados y los tres restantes fueron homogeneizados y congelados a $-20^{\circ} \mathrm{C}$, para el correspondiente análisis posterior. Estas muestras fueron de las variedades Perla (P1), Puquina (P2), India (P3), Chaposa (P4), Robusta (P5) y Jaspe (P6) que fueron cultivadas tanto en Morochata (Código M) como en Chullchunqani (Código C). Los parámetros analizados fueron, siguiendo con pocas variantes a los métodos recomendados de la AOAC (1): humedad, cenizas, grasa cruda, proteína cruda (con factor Nx6.25) y fibra dietética total (método enzimático). También fueron determinados el valor energético por bomba calorimétrica, el contenido de minerales por espectroscopia de emisión de plasma inducido, el contenido en glicoalcaloides totales según el método de Hellenas $(5,6)$ y el contenido en aminoácidos por cromatografía de intercambio iónico. Sacarosa, fructosa y glucosa fueron determinados por cromatografía líquida de alto rendimiento. El almidón fue separado previamente y luego de ser hidrolizado enzimáticamente fue calculado a partir de resultados de cromatografía líquida de alto rendimiento.

Finalmente se recogieron en la campaña del año 95/96 muestras de dos ensayos de cultivo de la variedad Robusta realizados en las localidades de Toralapa (altitud: 3,430 msnm, precipitación media anual: $530 \mathrm{~mm}$ y temperatura promedio anual: $11{ }^{\circ} \mathrm{C}$ ) y Mizque (altitud: $2,025 \mathrm{msnm}$, precipitación media anual: $508 \mathrm{~mm}$ y temperatura promedio anual: $19.8^{\circ} \mathrm{C}$ ). En ambos casos se recogieron 8 a 10 tubérculos de 4.5 a $6 \mathrm{~cm}$ de diámetro, y se realizaron los análisis por triplicado, siguiendo el método anteriormente indicado para determinar el contenido en glicoalcaloides, tanto en la parte comestible como en las cáscaras de cada caso.

\section{Diseño experimental}

Durante los tres primeros años las parcelas fueron establecidas en bloques simples sin repeticiones por el número elevado de clones con que se contaba, colocando 10 tubérculos de cada clon en un surco de $3 \mathrm{~m}$. A partir de la campaña 1992-1993, se usó el Diseño de Bloques Completos al Azar (14), con cuatro repeticiones y 30 tubérculos por clon, usando la misma metodología en ambas localidades (Tabla 2) 
Tabla 2. Número de clones evaluados por su resistencia al tizón durante cinco campañas agrícolas

\begin{tabular}{|cc|}
\hline Campaña & No. clones sembrados \\
\hline $1990-1991$ & 313 \\
$1991-1992$ & 170 \\
$1992-1993$ & 31 \\
$1993-1994$ & 15 \\
$1994-1995$ & 10 \\
\hline
\end{tabular}

Cada unidad experimental estuvo constituida por dos surcos de $4.50 \mathrm{~m}$ de longitud, espaciados a $0.70 \mathrm{~m}$, con distancia entre plantas de $0.30 \mathrm{~m}$. El área total del ensayo fue variable para cada una de las campañas, puesto que cada vez se reducía el número de tratamientos (clones).

\section{Análisis estadísticos}

Con las lecturas realizadas por daño de tizón se realizó el cálculo del AUDPC (Área Bajo la Curva de Progreso de P. infestans), para lo cual se usó la fórmula propuesta por Shaner y Finney (13), para así obtener un dato único y realizar el análisis estadístico.

$$
A U D P C=\sum_{i=1}^{n}\left[\left(X_{i+1}+X_{i}\right) / 2\right]\left(T_{i+1}-t i\right)
$$

Donde :

Xi $=\quad$ Proporción de tejido afectado en la observación

$\left(T_{i+1}-\mathrm{ti}\right)=$ Tiempo en dias entre dos lecturas

$\mathrm{n}=\quad$ Número total de observaciones

Con los datos obtenidos de este análisis y los rendimientos medidos se realizaron los análisis de variancia y comparaciones de medidas. 


\section{Resultados y Discusión}

\section{Daño por tizón tardío}

Durante las cinco campañas agrícolas, el grado de daño por tizón para cada uno de los clones fue variable, principalmente por las condiciones de humedad y temperatura reinantes en las localidades de prueba, lo que determinó la severidad del ataque de las diferentes poblaciones existentes en cada una de ellas.

En la Tabla 3 se muestra el grado de daño alcanzado por los seis clones promisorios que destacaron durante los años de evaluación. Además se observa que el grado de daño alcanzado por cada uno de los clones, es siempre menor que los testigos, a excepción de los años 1992/93 y 1994/95, en los que el testigo resistente, Rosita, mostró menor grado de daño que algunos de los clones (Tabla 5).

Tabla 3. Área bajo la curva de progreso de la enfermedad (AUDPC) de seis clones promisorios y tres testigos durante cinco campañas agrícolas.

\begin{tabular}{cccccc}
\hline & \multicolumn{5}{c}{ Campaña agrícola } \\
\cline { 2 - 6 } Clon & $1990 / 1991$ & $19991 / 1992$ & $1992 / 1993$ & $1993 / 1994$ & $1994 / 1995$ \\
\hline 575045 & 45.0 & 166.3 & 140.2 & 45.3 & 30.0 \\
720049 & 50.0 & 487.0 & 595.0 & 265.0 & 48.6 \\
676008 & 49.0 & 166.3 & 255.9 & 257.2 & 86.4 \\
385240.2 & 47.0 & 487.5 & 132.4 & 823.8 & 279.8 \\
$84-75-16$ & 31.5 & 68.8 & 265.3 & 1581.0 & 157.7 \\
$79-94-3$ & 49.0 & 35.0 & 48.1 & 110.0 & 561.7 \\
Waych'a & 601.3 & 1803.8 & 500.0 & 3294.0 & 2601.5 \\
Alpha & 986.5 & 2700.0 & 950.0 & 4293.0 & 3664.0 \\
Rosita & 542.5 & 750.0 & 458.0 & 1688.0 & 208.4 \\
\hline
\end{tabular}

Fuente: (8)-(12).

Para las tres últimas campañas se realizaron los análisis de variancia respectivos. En la Tabla 4 se muestra el análisis de la última campaña (1994/95), en la cual se procedió a la selección y nominación de seis cultivares potenciales y por considerarse la más representativa, esta misma campaña se tomó como referencia para analizar los rendimientos (Tabla 6). 
Tabla 4. Análisis de variancia para AUDPC de diez clones promisorios y tres testigos. (Campaña 1994/95, Cochabamba, Bolivia)

\begin{tabular}{lccccc}
\hline & & \multicolumn{2}{c}{ Morochata } & \multicolumn{2}{c}{ Chulchunqani } \\
\cline { 3 - 6 } F.V. & G.L & C.M & F & C.M & F \\
\hline Repeticiones & 3 & 22735.419 & $1.16^{* *}$ & 14241.065 & $1.08^{* *}$ \\
Tratamientos & 12 & 5460289.434 & $277.82^{* *}$ & 5317843.730 & $403.57^{* *}$ \\
Error & 36 & 19654.170 & & 13176.852 & \\
\hline Total & 51 & & \\
\hline & & C.V. $=17.34 \%$ & C.V. $=17.16 \%$
\end{tabular}

El análisis señaló diferencias altamente significativas entre tratamientos para las dos localidades; diferencias atribuibles principalmente a las existentes entre clones y testigos, lo que ratificaría la resistencia de los clones al tizón, observada en campañas anteriores.

\section{Rendimiento}

La resistencia observada en follaje debe ser confirmada con los rendimientos obtenidos y atributos agronómicos de los tubérculos para de esta manera proceder a la selección de aquellos sobresalientes en todos estos aspectos. Los rendimientos obtenidos durante las cinco campañas de evaluación por los seis clones (Tabla 5), fueron superiores a los testigos e inclusive al testigo resistente. Estos rendimientos estarían en un rango de 30 a 56 t/ha; rendimientos muy superiores a los obtenidos actualmente, que en el mejor de los casos alcanza a 25 t/ha con una alta inversión en fungicidas.

El análisis de variancia para rendimientos (Tabla 6), mostró también diferencias altamente significativas en ambas localidades, aunque también existen diferencias entre ellos. 
Tabla 5. Rendimiento en kg/planta de seis clones promisorios y tres testigos durante cinco campañas agrícolas, en dos localidades de prueba.

\begin{tabular}{cccccc}
\hline & \multicolumn{5}{c}{ Campaña agrícola } \\
\cline { 2 - 6 } Clon & $1990 / 1991$ & $1991 / 1992$ & $1992 / 1993^{*}$ & $1993 / 1994^{*}$ & $1994 / 1995^{*}$ \\
\hline 575045 & 0.93 & 0.90 & 1.19 & 1.20 & 1.16 \\
720049 & 0.91 & 0.82 & 1.13 & 052 & 0.80 \\
676008 & 1.10 & 0.65 & 1.09 & 0.80 & 0.82 \\
385240.2 & 0.95 & 0.88 & 1.07 & 0.72 & 0.85 \\
$84-75-16$ & 1.18 & 0.69 & 0.81 & 0.63 & 0.81 \\
$79-94-3$ & 0.76 & 0.99 & 1.00 & 1.03 & 0.69 \\
Waych'a & 0.46 & 0.15 & 0.54 & 0.07 & 0.23 \\
Alpha & 0.52 & 0.19 & 0.54 & 0.06 & 0.18 \\
Rosita & 0.65 & 0.47 & 0.77 & 0.11 & 0.52 \\
\hline
\end{tabular}

* Promedio de cuatro repeticiones.

Tabla 6. $\quad$ Análisis de variancia para rendimiento de diez clones promisorios $y$ tres testigos. (Campaña 1994/95, Cochabamba, Bolivia).

\begin{tabular}{lccccc}
\hline & & \multicolumn{2}{c}{ Morochata } & \multicolumn{2}{c}{ Chullchunqani } \\
\cline { 3 - 6 } \multicolumn{1}{c}{ F.V. } & G.L. & C.M. & F & C.M & F \\
\hline Repeticiones & 3 & 0.022 & 1.26 & 0.026 & 3.11 \\
Tratamientos & 12 & 0.569 & $32.70^{\star \star}$ & 0.221 & 26.23 ** \\
Error & 36 & 0.017 & & 0.008 & \\
\hline Total & 51 & & & \\
\hline & & C.V. $=16.35 \%$ & & C.V. $=17.33 \%$
\end{tabular}

Durante la última campaña se estudiaron 10 clones con sus respectivos testigos de los cuales destacan algunos en su resistencia al tizón y rendimientos como se puede observar en la Figura 1.

Las evaluaciones realizadas y principalmente las evaluaciones de grado de daño, nos indicarían que los clones tienen resistencia combinada (específica y general), la cual es compleja de definir. 


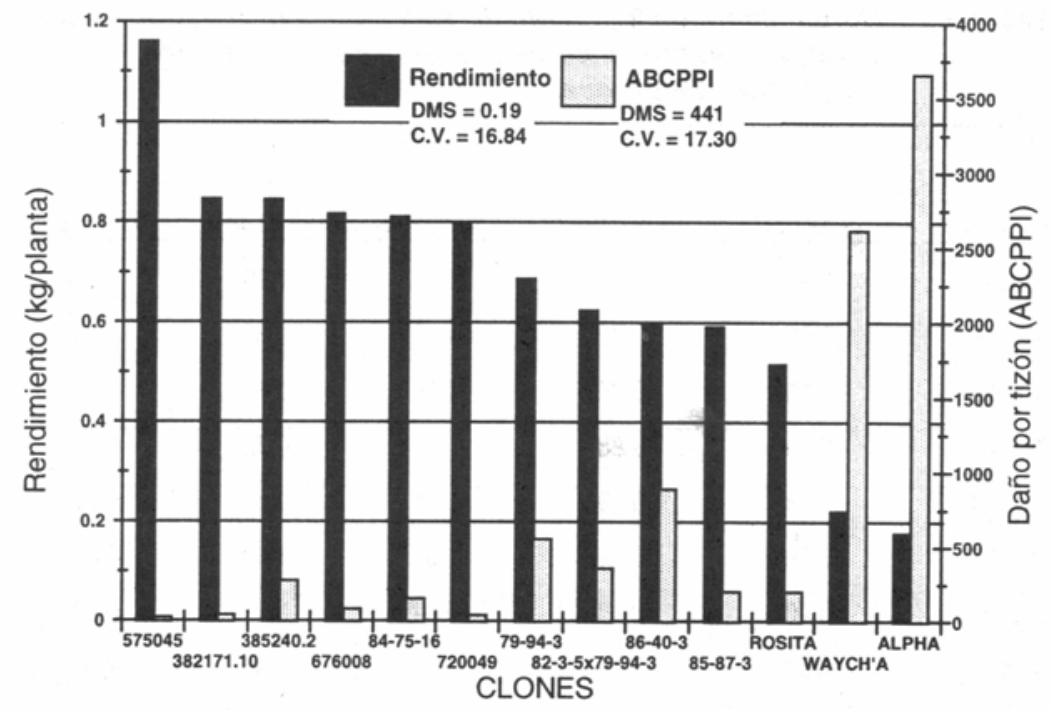

Figura 1. Efecto del daño por tizón (AUDPC) sobre el rendimiento de diez clones promisorios resistentes al tizón de la papa y tres cultivares testigo, promedio de cuatro repeticiones por localidad $y$ dos localidades. (Morochata-Chullchunqani 94-95).

\section{Evaluación de poscosecha}

Luego de todas las observaciones agronómicas, en la última evaluación se efectuaron pruebas de degustación con agricultores y los análisis correspondientes a la composición química y el contenido de glicoalcaloides.

En las evaluaciones de degustación con agricultores, aun cuando todos los clones estuvieron dentro del rango aceptable (regular-bueno), destacó notablemente el clon 676008 alcanzando un puntaje similar al testigo (Tabla 7). 
Tabla 7. Calificación promedio obtenida en pruebas de degustación con agricultores. (Morochata-Chullchunqani 1994/95).

\begin{tabular}{lccc}
\hline \multirow{2}{*}{ Clon } & \multicolumn{2}{c}{ LOCALIDAD } & \\
\cline { 2 - 3 } & $\begin{array}{c}\text { Morochata } \\
\text { Promedio* }\end{array}$ & $\begin{array}{c}\text { Chullchunqani } \\
\text { Promedio** }\end{array}$ & $\begin{array}{c}\text { Promedio } \\
\text { General }\end{array}$ \\
\hline CIP-575045 & 2.3 & 2.2 & 2.3 \\
CIP-720049 & 2.4 & 2.6 & 2.5 \\
CIP-676008 & 2.9 & 2.3 & 2.6 \\
CIP-385240.2 & 2.0 & 2.4 & 2.2 \\
ICA-84-75-16 & 2.4 & 1.7 & 2.1 \\
ICA-79-94-3 & 2.4 & 2.4 & 2.4 \\
Waych'a & 2.9 & 2.3 & 2.6 \\
\hline
\end{tabular}

* Promedio de cuatro comunidades.

** Promedio de dos comunidades.

\section{Entrega de cultivares potenciales}

Luego de cinco años de evaluación y las pruebas realizadas, se procedió a entregar a los agricultores los seis mejores clones, como cultivares potenciales con los nombres y características que se detallan en la Tabla 8.

Los cultivares que se muestran en la Tabla 8, siguen siendo evaluados bajo diferentes condiciones de producción y principalmente bajo el manejo de agricultores e instituciones semilleristas y productores de papa, para que finalmente puedan demandar la semilla de aquellos cultivares que mejor se adapten a sus condiciones. Además, se realizarán pruebas de evaluación con otros usuarios como los intermediarios, consumidores y plantas de procesamiento.

Los resultados promedio de los análisis químicos realizados en las muestras de papas de las variedades promisorias de la campaña del 94/ 95 muestran que la composición química es similar a los datos generales reportados en la literatura (15). Sin embargo se encuentran resultados con una variación muy importante en el contenido de glicoalcaloides por variedad, tal como se pueden observar en la Tabla 9. Los valores del contenido de glicoalcaloides de la Tabla 9 están expresados en base seca y ello permite una comparación entre todos. Es evidente que el tamaño de muestra influye sobre los resultados; sin embargo, se observa que las variedades Perla, Chaposa y Robusta presentan en general 
los mayores contenidos de glicoalcaloides. Todos los valores encontrados en las papas enteras y expresados en base húmeda, como se suele presentar los resultados de contenido de glicoalcaloides en papas, se encuentran en niveles por debajo de los 200 ppm, por lo que aún las variedades Perla, Chaposa y Robusta no pueden considerarse como papas no aptas para el consumo. Pero en base a esta información y evaluaciones de agricultores, la variedad Chaposa ha sido puesta en observación por sus niveles elevados de glicoalcaloides.

Tabla 8. Principales características de los clones con resistencia al tizón de la papa (P. infestans) liberados en Cochabamba-Bolivia (1994-1995)

\begin{tabular}{|c|c|c|c|c|}
\hline Clon & Nombre & Pedigrí & $\begin{array}{l}\text { Características } \\
\text { principales }\end{array}$ & $\begin{array}{l}\text { Reacción a otros } \\
\text { factores }\end{array}$ \\
\hline CIP-575045 & Perla & $\begin{array}{l}\text { POOS-16 (tbr } \\
\text { Holanda) }\end{array}$ & $\begin{array}{l}\text { Tub.redondeados, } \\
\text { ojos superf., piel } \\
\text { crema, carne crema }\end{array}$ & $\begin{array}{l}\text { (MF) -Tolerante } \\
\text { (H)-Tolerante }\end{array}$ \\
\hline CIP-720049 & Puquina & $\begin{array}{l}\text { Furore }^{\star}(\text { US } 129.2 \\
\text { Kathadin) }\end{array}$ & $\begin{array}{l}\text { Tub.redondeados, } \\
\text { ojos superf., piel } \\
\text { rosada, carne crema }\end{array}$ & MF-Tolerante \\
\hline CIP-676008 & India & $\begin{array}{l}\text { US136.6*(3345D } \\
\left.(1)^{\star} 2288 \mathrm{~A}(2)\right)\end{array}$ & $\begin{array}{l}\text { Tub.redondeados, } \\
\text { ojos medianos, piel } \\
\text { rosada, carne crema }\end{array}$ & $\begin{array}{l}\text { MF-Tolerante } \\
(\mathrm{V}) \text { - } \\
\text { Moderadamente } \\
\text { resistente }\end{array}$ \\
\hline CIP-385240.2 & Chaposa & \begin{tabular}{|l} 
AVRDC- \\
$1287.19^{\star}(\mathrm{BL}-2) 9$
\end{tabular} & $\begin{array}{l}\text { Tub.redondeados, } \\
\text { ojos medianos, } \\
\text { carne crema halo } \\
\text { rosado, carne crema }\end{array}$ & MF-Tolerante \\
\hline ICA-84-75-16 & Jaspe & $\left(\right.$ sto*bre ${ }^{\star}\left(\right.$ tbr $^{\star}$ adg) & $\begin{array}{l}\text { Tub.redondeados, } \\
\text { ojos mediano, piel } \\
\text { crema halo morado, } \\
\text { carne crema } \\
\end{array}$ & $\begin{array}{l}\text { (A)-Resistente } \\
\text { (S)-Tolerante }\end{array}$ \\
\hline ICA-79-94-3 & Robusta & $\left(\mathrm{tbr}^{\star} \mathrm{adg}\right)^{\star} \mathrm{tbr}$ & $\begin{array}{l}\text { Tub.comprimido, } \\
\text { ojos medianos, piel } \\
\text { rosado-crema, carne } \\
\text { crema }\end{array}$ & $\begin{array}{l}\text { (MF)-Tolerante } \\
\text { (V)-Resistente }\end{array}$ \\
\hline
\end{tabular}

Fuente: (2)

1. (MF): manchas foliares; $(H)$ : heladas; $(V)$ :verruga; $(N)$ : Naccobus; (S): sequía 
Tabla 9. Contenido de glicoalcaloides totales (ppm en base seca) en las variedades Perla (P1), Puquina (P2), India (P3), Chaposa (P4), Robusta (P5), y Jaspe (P6), cultivadas en Chullchunqani y Morochata.

\begin{tabular}{lllllllllllllll}
\hline \multicolumn{10}{c}{ Código de la muestra } \\
\hline $\begin{array}{l}\text { Contenido de } \\
\text { glicoalcaloides } \\
\text { totales }\end{array}$ & P1C* & P2C & P3C & P4C & P5C & P6C & P1M* & P2M & P3M & P4M & P5M & P6M \\
\hline $\begin{array}{l}\text { Parte } \\
\begin{array}{l}\text { comestible } \\
\text { liofilizada }\end{array}\end{array}$ & 1035.8 & 78.7 & 98.0 & 371.6 & 356.4 & 17.4 & 375.6 & 33.0 & 38.5 & 572.5 & 271.7 & 22.6 \\
\hline
\end{tabular}

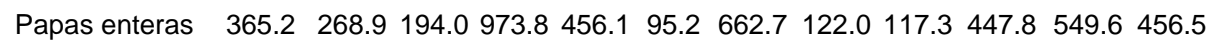

$$
\mathrm{C}=\text { Chullchunqani } \mathrm{M}=\text { Morochata }
$$

Respecto al contenido de solanina y chaconina en tubérculos y cáscaras de la variedad Robusta producida en las localidades de Toralapa y Mizque hubo variación considerable con el lugar de cultivo (Tabla 10). En efecto, entre los valores de glicoalcaloides obtenidos para la parte comestible y cáscaras de los tubérculos de la variedad Robusta cultivada en Toralapa y en Mizque hubo variaciones considerables. Por otra parte, las proporciones relativas de chaconina y solanina de los tubérculos presentaron variación con la variedad pero no con el lugar de cultivo. Estos resultados demuestran la importancia de determinar los niveles en glicoalcaloides en las nuevas variedades bajo diferentes condiciones agroclimáticas para asegurar que estos niveles se queden dentro del margen aceptable.

Tabla 10. Contenido de Solanina y Chaconina de tubérculos de la variedad Robusta cultivada en Toralapa y Mizque (expresados en ppm sobre materia seca; valor medio +/-desviación estándar).

\begin{tabular}{|lllll|}
\hline \multirow{2}{*}{$\begin{array}{l}\text { Lugar de } \\
\text { cultivo }\end{array}$} & \multicolumn{2}{c}{ Parte comestible } & \multicolumn{2}{c|}{ Cáscaras } \\
\cline { 2 - 5 } $\begin{array}{l}\text { Toralapa } \\
\text { Mizque }\end{array}$ & $26.2+/-3.4$ & Chaconina & Solanina & Chaconina \\
Mizuna & $270.8+/-102.5$ & $555.9+/-181.8$ & $1139.8+/-53.4$ & $2237+/-206.0$ \\
\hline
\end{tabular}




\section{Conclusiones}

- Se entregaron seis cultivares potenciales con resistencia al tizón tardío ( $P$. infestans), con rendimientos superiores a los cultivares en actual uso, buenas características agronómicas, calidad culinaria aceptable y que en la actualidad están en evaluación en diferentes condiciones de producción en campos de agricultores y con otros usuarios potenciales.

- Estos nuevos cultivares poseen resistencia combinada, es decir poseen resistencia o tolerancia a factores bióticos y abióticos, lo que los hace aún más ventajosos.

- Estos cultivares formarán parte de un conjunto de prácticas de manejo integrado para el control del tizón para reducir el número de aplicaciones de fungicidas significativamente.

- Los rendimientos más altos y el reducido número de aplicaciones de pesticidas, permitirán mejorar el ingreso económico de los agricultores.

- Los contenidos de glicoalcaloides en los cultivares potenciales, están dentro del límite permitido para consumo humano (20 $\mathrm{mg} / 100 \mathrm{~g}$ de muestra fresca), a excepción del cultivar Chaposa, el cual sobrepasa este límite.

- El análisis de glicoalcaloides debe realizarse bajo diferentes condiciones agroclimáticas considerando la variación en concentración observada de acuerdo al lugar de muestreo.

- Se necesita más investigación sobre la relación entre la concentración de glicoalcaloides y las condiciones agroclimáticas.

Los autores expresan su más sincero agradecimiento al Programa Cooperativo de Investigación en Papa (PRACIPA), por su contribución económica en buena parte de los trabajos desarrollados.

También dejamos expresa constancia de nuestro agradecimiento al Ing. Rudy Torrez y al Sr. Jorge Bustamante, investigadores de PROINPA, quienes colaboraron ampliamente en las pruebas de calidad culinaria 


\section{Referencias Bibliográficas}

1. A.O.A.C.15ª Edición, 1990, EE.UU..

2. Carrasco, E; Estrada, N; Gabriel, J; Quiroga, O; García, W; Mendoza, O. 1995. Seis nuevas variedades de papa con resistencia al tizón (Phytophthora infestans). Boletín Técnico Instituto Boliviano de Tecnología Agropecuaria, TECNO-IBTA 1 (5): 1-8.

3. Estrada, N.; Pérez-Alvarez, O.; Henfling, J.; Malamud, O. 1983. In Research for the Potato in the Year 2000. W.J. Hooker, (ed). p. 78-79. International Potato Center, Lima-Perú.

4. Estrada, N.; Fernández-Northcote, E.; Carrasco, E.; Navia, O. 1994. Mejoramiento genético para resistencia a enfermedades y plagas de la papa en Bolivia. En: Memorias del 1er Taller sobre Resistencia Duradera en Cultivos Alto Andinos de Bolivia, Colombia, Ecuador y Perú. Quito-Ecuador. 111 p.

5. Hellenas, K.E. 1986. A simplified procedure for quantification of potatoglycoalkaloids in tuber extracts by HPLC; comparison with ELISA and a colorimetric method, J. Sci. Food Agric. 37:776-782.

6. Hellenas K.E.; Branzell, C.; Johnsson, H.; Slanine, P. 1995 Glycoalkaloid content of early potato varieties. J. Sci. Food Agric. 67:125-128.

7. Linzer, A.K. 1995. El diagnóstico rural participativo un método para la planificación de proyectos con comunidades rurales. Manual para el sistema regional de transferencia de tecnología agropecuaria. Módulo 11. Centro de Investigación Agrícola Tropical (CIAT). Santa Cruz-Bolivia. p. 88.

8. Programa de Investigación de la Papa (PROINPA). 1991. Informe Anual PROINPA, 1990-1991. Cochabamba, Bolivia. 260 p.

9. Programa de Investigación de la Papa (PROINPA). 1992. Informe Anual PROINPA, 1991-1992. Cochabamba, Bolivia. 297 p.

10. Programa de Investigación de la Papa (PROINPA). 1993. Informe Anual PROINPA, 1992-1993. Cochabamba, Bolivia. 483 p.

11. Programa de Investigación de la Papa (PROINPA). 1994. Informe Anual PROINPA, 1993-1994. Cochabamba, Bolivia. 620 p. 
12. Programa de Investigación de la Papa (PROINPA). 1995. Informe Anual PROINPA, 1994-1995. Cochabamba, Bolivia. 900 p.

13. Shaner, G; Finney, R.E. 1977. The effect of nitrogen fertilization on the expression of slow-mildewing resistance in knox wheat. Phytopathology 67:1051-1056.

14. Steel, R. G.; Torrie, J.H. 1990. Bioestadística: Principios y procedimientos. Segunda edición, primera en español. Mc GrawHill. México. 619 p.

15. Woofe, J. A. 1987. Potato in the human diet. Cambridge University Press. Great Britain. 231 p. 